\title{
MATRIZ ORIGEN-DESTINO Y EFICIENCIA EN MODOS DE TRANSPORTE URBANO: UN ANÁLISIS DE LA MOVILIDAD DE BOGOTÁ*
}

Recibido: 21 de julio de 2015 - Aprobado: 17 de febrero de 2016

\author{
Laura L. Amézquita** \\ Duván F. Durán Matiz ${ }^{* * *}$ \\ Danny Heriberth Fajardo Morales ${ }^{* * * *}$
}

\section{RESUMEN}

Este artículo presenta un análisis de la movilidad en Bogotá. Se estudia la generación de externalidades negativas del transporte público colectivo frente a otros modos de transportes alternativos que son sustitutos como el Sistema de Transporte Masivo Transmilenio. A partir de la encuesta de movilidad 2011 se construyen matrices origen-destino y se explora la eficiencia en los desplazamientos a través de los tiempos de duración de viaje en la ciudad en función de las distancias y de los modos de transporte. Se concluye, a partir de estimaciones econométricas, que la duración de tiempos de viajes se ve afectada por las distancias recorridas en forma negativa y el modo de transporte (transporte público colectivo y/o Transmilenio) de forma indistinta.

\section{PALABRAS CLAVE}

Movilidad, matriz origen-destino, Bogotá

\section{CLASIFICACIÓN JEL}

R41, R12, R58

\section{CONTENIDO}

Introducción; 1. Problemas de movilidad en ciencia económica; 2. La movilidad en Bogotá D.C; 3. Metodología y análisis de resultados; 4. Conclusiones y consideraciones finales; Bibliografía; Anexos.

* Este artículo de investigación es producto del Proyecto "Economía y Territorio (2010-2016)" y hace parte del trabajo del grupo de investigación en economía y desarrollo humano, de la Universidad de La Salle, clasificado por Colciencias en categoría B. Resultados preliminares fueron presentados en la monografía de grado "Estudio del transporte público en Bogotá 2011". Los datos fueron obtenidos a través del convenio de asociación No 21/2013 entre la Universidad de La Salle y la Secretaría Distrital de Planeación de Bogotá.

** Economista, Universidad de los Andes, Bogotá, Colombia. Politóloga Universidad de los Andes, Bogotá, Colombia. Máster en Urbanismo y Planificación del Territorio, Université Paris 1, Panthéon-Sorbonne, Paris, Francia. Candidata a doctora en Ciencias Humanas Université de Neuchâtel, Neuchâtel, Suiza. Groupe de Recherche en Économie Territoriale (GRET) Université de Neuchâtel, Suiza. Grupo de investigación en economía y desarrollo humano, Universidad de La Salle, Bogotá, Colombia. Dirección postal: Carrera 5 No 59A-44 Edificio de postgrados Hno. Justo Ramón 5to piso. Teléfono +57 1 4641790. Correo electrónico: laura.amezquita@unine.ch.

*** Economista, Universidad de La Salle, Bogotá, Colombia. Integrante del grupo de investigación en economía y desarrollo humano, Universidad De La Salle, Bogotá, Colombia. Dirección postal: Carrera 21 No 36-17 sur Bogotá, Colombia. Teléfono: +57 3142945134, +573004700799. Correo electrónico: dduran07@ unisalle.edu.co.

**** Economista, Universidad de La Salle, Bogotá, Colombia. Integrante del grupo de investigación en economía y desarrollo humano, Universidad de La Salle, Bogotá, Colombia. Calle 159 \# 19a-20 Apto 508, Bogotá, Colombia. Teléfono:+57 1 7575588, +57 3106743221. Correo electrónico: dfajardo27@unisalle.edu.co. 


\section{ORIGIN-DESTINATION MATRIX AND EFFICIENCY IN URBAN MEANS OF TRANSPORTATION: BOGOTA'S MOBILITY ANALYSIS}

\section{ABSTRACT}

This article analyses mobility in Bogota. It studies the generation of negative externalities of Collective Public Transportation towards other means of substitute alternative transportation such as the Transmilenio Transportation System. Using the 2011 mobility survey, origin-destination matrixes are built while exploring the efficiency in displacements by travel times within the city in function to distances and means of transport. Parting from econometric estimations It is concluded that the duration of displacements is affected by the negatively covered distances and the mean of transportation (Collective Public Transportation and / or Transmilenio) indistinctively.

\section{KEY WORDS}

Mobility, Origin - Destination Matrix, Bogota

\section{JEL CLASSIFICATION}

R41, R12, R58

\section{CONTENT}

Introduction; 1. Mobility problems in economics; 2. Mobility in Bogota D. C.; 3. Methodology and analysis of results; Conclusions and final considerations; Bibliography.

\section{MATRIZ ORIGEM DESTINO E EFICIÊNCIA EM MODOS DE TRANSPORTEURBANO: UMA ANÁLISE DA MOBILIDADE EM BOGOTÁ RESUMO}

Este artigo apresenta uma análise da mobilidade em Bogotá. Se estuda a geração de externalidades negativas do Transporte Público Coletivo frente a outros modos de transportes alternativos que são substitutos como o Sistema de Transporte Massivo Transmilenio. A partir da enquete de mobilidade 2011 se construíram matrizes origem destino e se explora a eficiência nos deslocamentos através dos tempos de duração da viagem na cidade em função das distâncias e dos modos de transporte. Se conclui, a partir de estimações econométricas que a duração de tempos de viagens se vê afetada pelas distâncias recorridas em forma negativa e o modo de transporte (Transporte Público Coletivo e/ou Transmilenio) de forma indistinta.

\section{PALAVRAS CHAVE}

Mobilidade, Matriz Origem-Destino, Bogotá

\section{CLASSIFICAÇÃO JEL}

R41, R12, R58

\section{CONTEÚDO}

Introdução; 1. Problemas de mobilidade em ciência económica; 2. A mobilidade em Bogotá D.C; 3. Metodologia e análise de resultados; 4. Conclusões e considerações finais; Bibliografia; Anexos. 


\section{INTRODUCCIÓN}

La movilidad de las ciudades contribuye a su consolidación, a crear un sistema económico cada vez más globalizado y competitivo en busca de mejores condiciones de vida y facilidades para la ejecución de actividades productivas de la población, como lo plantean Alcântara (2010), Camagni (2005) y Matas (2004). La comprensión de los sistemas de movilidad podría tener efectos sobre la eficiencia de la economía urbana.

El transporte público colectivo (en adelante TPC) representa una de las diferentes alternativas de desplazamientos, entre los cuales se encuentran los modos como buses de tránsito rápido (BRT ${ }^{1}$, en Bogotá el Sistema Transmilenio), la bicicleta, el automóvil particular o la marcha a pie, entre otros. La calidad de los medios de transporte en el contexto urbano indica las condiciones a las que se enfrenta el ciudadano común en el disfrute y acceso a los servicios urbanos.

\section{Como lo menciona la Alcaldía Mayor de Bogotá (s.f.):}

La inequidad inexistente entre los diferentes modos de transporte respecto a la relación del uso de la infraestructura [...], ha generado la saturación de las vías primarias y por consiguiente que la velocidad de desplazamiento se haya reducido, especialmente durante los periodos pico. Lo anterior indica un bajo nivel de servicio que se traduce en un mayor tiempo de recorrido en los desplazamientos, en una gran pérdida de horas-hombre ocupados en el tráfico, con mayores consumos de combustibles e importantes niveles de contaminación al medio ambiente derivados de la baja velocidad.

En el caso de urbes, como Bogotá, es posible que el objetivo principal de moverse de un punto a otro se vea obstaculizado por diferentes aspectos en su operación, es decir, que se involucren temas relacionados con largos tiempos de viaje, baja calidad del servicio, sobreoferta de vehículos (congestión vehicular), parque automotor obsoleto, contaminación, entre otros, que dificultan el desarrollo de un sistema de movilidad eficiente e incrementan los altos costos de transacción, para la ciudad en general. De igual manera se presentan intereses que involucran al sector privado, permeados por la dinámica de mercado en la que los empresarios buscan aumentar el número de vehículos con el fin de obtener mayores ganancias. En este contexto el TPC para Bogotá se constituye como uno de los desafíos a resolver en cuanto al mejoramiento del sistema por la operación de buses (44\%), microbuses (32\%), y busetas (24 \%) que conforman el TPC, de acuerdo con el Informe de Movilidad 2011 de Steer Davies Gleave (2012).

1 Bus de tránsito rápido (BRT por sus siglas en inglés Bus Rapid Transit) 
El presente artículo indaga sobre el estado de los desplazamientos realizados en Bogotá, a partir de la encuesta de movilidad de 2011. Propone la caracterización de la movilidad cotidiana en uso de matrices origen-destino, metodología que no ha sido trabajada al nivel de localidades y en relación con los 17 municipios de la Zona Metropolitana de Bogotá que cubre la encuesta², así como la cartografía correspondiente. Además, se propone un sencillo modelo de regresión que indaga la eficiencia en modos de transporte (TPC frente a Transmilenio) en términos de tiempos de los desplazamientos.

Luego de esta introducción, se presenta un breve marco teórico para los problemas de movilidad desde la ciencia económica; en la tercera parte se expone una lectura sobre el estado actual de la movilidad en Bogotá; en la cuarta parte, la metodología y los resultados, y, por último, unas conclusiones y consideraciones finales.

\section{PROBLEMAS DE MOVILIDAD EN CIENCIA ECONÓMICA}

La movilidad de la población en el espacio urbano permite materializar la relación entre los diferentes roles de cada individuo, tales como: estilos de vida en un contexto de división funcional en las unidades familiares, actividades y espacios cotidianos, división funcional tanto del espacio como de la estructura urbana y metropolitana como lo reseñan Díaz y Jiménez (2002) y Moro (2007). La movilidad acá se concibe como un medio a través del cual se permite un desplazamiento de un lugar a otro con un objetivo en específico, donde se reconoce el desarrollo de las diferentes actividades de la sociedad enmarcada en un contexto local.

La teoría de la localización afirma que las personas se sitúan en función de las actividades productivas; en este sentido la configuración espacial incide en el proceso de producción y afecta el valor de los bienes. Siguiendo a Alonso (1964), Fujita, Krugman y Venables (1999) y Camagni (2005), como lo cita Duch (s. f.), los avances fundamentales en el análisis sobre la localización, incorporados a la teoría económica, vienen de la época de Marshall (alrededor de 1890) cuando se abordó el aspecto monetario en cuanto a las ventajas de la localización y la relación entre el coste del transporte y la distancia al centro de producción. Von Thünen (alrededor 1826) desarrolló el primer modelo formal de la localización en la actividad económica donde el principal aporte según Duch (s. f., p. 10) "a la teoría de la localización fue el reconocimiento de que la distancia, y por tanto los costes de transporte, imponen un ordenamiento espacial de las actividades económicas".

2 Es preciso aclarar que, después de realizada la indagación dentro del presente trabajo investigativo, la SDP (2014) publicó el libro "Región Metropolitana de Bogotá: Una visión de la ocupación del suelo" donde presenta matrices origen destino cuya unidad de análisis ha sido la zona metropolitana, por tanto, estudia los 17 municipios de la encuesta utilizando el método de imputación. 
Matriz origen-destino y eficiencia en modos de transporte urbano: un análisis de la movilidad de Bogotá

Desde el enfoque clásico, De Rus, Campos y Nombela (2003, p. 18) exponen que:

[... en el modelo tradicional de competencia perfecta, los efectos de una inversión en una infraestructura que disminuye el coste de transporte son bien conocidos: reducción del coste marginal, beneficios extraordinarios en el corto plazo, entrada de nuevas empresas y nuevo equilibrio en el largo plazo con un precio menor y mayor nivel de producción. Los consumidores son los beneficiarios finales de la disminución de los costes de transporte.

La economía del transporte estudia los cambios en los factores de innovación que se relacionan con procesos de administración, planificación y ejecución de recursos para la construcción de infraestructura de transporte. En este sentido, profundiza en los "efectos económicos de los movimientos de personas y mercancías, y es evidente que para facilitar dichos movimientos es preciso contar con una adecuada dotación de infraestructuras de transporte", como lo anuncian Analistas Económicos de Andalucia, (s. f., p. 17). Thompson (1976) plantea que el aumento de las posibilidades culturales y sociales de las personas se debe a la generación de medios de transporte y al desarrollo de infraestructuras que comunican los lugares de trabajo con las residencias.

De otro lado, la nueva economía institucional (NEI) es una corriente que toma mayor relevancia a mediados de los años setenta, en oposición a planteamientos abstractos e individualistas de la economía neoclásica y la teoría de la localización. Esto constituye un replanteamiento de la ideología presentada por su predecesora escuela institucionalista retomando conceptos tales como los costos de transacción y las organizaciones con base en los planteamientos de Carrasco y Castaño (2012). En este sentido, la corrección de externalidades negativas que generan mayores costos de transacción en los sistemas de movilidad (ineficientes) es objeto de estudio de la NEI. Las instituciones representan las reglas de juego que logran establecer límites en el actuar de los individuos y moldean la forma en la que evoluciona la interacción humana. De esta manera, las instituciones se convierten en una estructura que permite condicionar la vida de los individuos, reduciendo la incertidumbre, justamente por el conocimiento de los comportamientos que rigen los individuos en una sociedad. Siguiendo a North (1990, p. 16) "Las instituciones afectan el desempeño de la economía debido a su efecto sobre los costos del cambio de la producción. Junto con la tecnología empleada determinan los costos de transacción y transformación (producción) que constituyen los costos totales". El éxito de las instituciones depende de la correcta formulación, aplicación y monitorización por parte de las autoridades pertinentes. El punto de partida en el presente análisis es la autoridad local, ya que es aquella quien puede intervenir el territorio con el fin de lograr corregir las externalidades. 


\section{LA MOVILIDAD EN BOGOTÁ}

Alcántara (2010, p. 191) hace una revisión detallada de cada uno de los componentes físicos y de la teoría de la movilidad urbana:

La metodología tradicional de análisis de la movilidad tiene un carácter eminentemente técnico, limitándose a cuantificar los desplazamientos. Dicha metodología parte del supuesto de que la movilidad es un "dato", que surge del desarrollo natural de la sociedad y que, por lo tanto, no debe ser discutida. Con ello se inhibe el análisis social y político de la movilidad para conocer quienes se movilizan y cómo, cuáles son las consecuencias para las personas y cómo son distribuidos los costos y los beneficios.

Un aspecto a considerar en este estudio es que las maneras de acceder a las alternativas de trasporte público de una ciudad tienen que ver con el ingreso de la población; según Matas (2004, p. 63), "el precio puede ser el mecanismo básico que garantice un uso eficiente de la infraestructura". La movilidad es un problema complejo que está vigente en las etapas de crecimiento de la ciudad, y por esto se constituye como unos de los principales ejes de la política de planeación distrital de acuerdo con Rodríguez (1990) y Moro (2007). Uno de los componentes en la eficiencia se da por el tiempo gastado en el movilizarse, esto estrechamente ligado a la cobertura de cada uno de los medios de transporte.

Bajo esta preocupación y para la ciudad de Bogotá, el problema ha sido tratado por diferentes autores. Hernández y Beltrán (2010) realizan un análisis del uso del servicio de transporte masivo en las localidades bogotanas de Ciudad Bolívar y Usme, examinando población de bajos recursos económicos (ingresos no superiores a dos salarios legales mínimos mensuales vigentes). Sus resultados indican que el rubro destinado al transporte es elevado frente a otros grupos de población con mayores niveles de ingreso, y que por este motivo es necesario ampliar la cobertura del servicio de Transmilenio en pro de las zonas de estudio.

El TPC y el Transmilenio constituyen la forma de desplazamiento masivo de personas en Bogotá; su característica principal es facilitar la movilidad de pasajeros por los diferentes corredores viales, con el cobro de una tarifa por la prestación del servicio. Para Rojas y Mello (2005, p. 108) "Esto se da a través de uno o varios modos de transporte, integrados o no, que se complementan y cuando el conjunto de partes que lo conforman interactúan entre sí por un objetivo común, existe un sistema de TPC". Para el caso de la ciudad de Bogotá, según Trasmilenio (2013) el TPC representa la forma de transporte tradicional, la cual ha permitido el desplazamiento de los ciudadanos desde la década de 1960 hasta la década de1990 tras la desaparición del tranvía y el ferrocarril a mediados del siglo XX. La prestación de este servicio se ha brindado 
a través de empresas privadas con ánimo de lucro, cuya operación es autorizada por la Secretaría Distrital de Movilidad. Se cuenta con un conjunto de vehículos representados en buses, busetas y microbuses para la prestación del servicio. Los actores que intervienen en este proceso están representados por empresarios, propietarios, conductores, usuarios e inclusive las mismas autoridades gubernamentales, con el fin de facilitar la operación del sistema a través de la regulación, monitorización y garantías en la prestación del servicio. Cada uno de estos actores mantiene sus propios intereses con base en sus necesidades.

El Concejo de Bogotá, mediante Acuerdo 04 de 1999, desarrolló un modo de transporte alternativo conocido como Transmilenio (Transmilenio 2013), justificado por los problemas de movilidad presentados desde la década de 1960 debido al incremento de la población y a la falta de interés político en la idea de movilidad eficiente. En paralelo el transporte masivo está en manos de empresarios privados que han provocado que la actividad sea de "manera anárquica, incomoda, costosa e insostenible", según Transmilenio (2013). Desde su creación los buses articulados y biarticulados son operados bajo el esquema BRT, han contado con una malla vial exclusiva y estaciones, y una flota complementaria de buses alimentadores. Estas características les han permitido tener complementariedad; contrario a ello, el TPC presenta una competencia entre sus prestadores de servicio de forma ineficaz, y no ha presentado cambios en los últimos 50 años, condiciones estas que señalan un bajo ritmo de evolución con respecto a las grandes trasformaciones que se generan en la ciudad, es decir, que el modelo de transporte no ha presentado innovaciones al mismo ritmo de la ciudad.

A continuación, se presenta (tabla 1) la distribución de los vehículos existentes de TPC que llevan a cabo la ejecución con y sin tarjeta de operación ${ }^{3}$ (TO) vigente. Se evidencia una participación total de vehículos con TO de 14.694, y para 2012, de 12.618 lo que representa una disminución del 16,4 \% de los vehículos totales, debido, en parte, a la implementación de un nuevo sistema que integra los diferentes modos de transporte en la ciudad (SITP).

La variación porcentual de un año a otro fue negativa, lo que quiere decir que el parque automotor del TPC tuvo una disminución en todos los tipos de transporte. En cuanto a los vehículos de TPC que sí tienen TO, entre 2011 y 2012 hubo una disminución considerable de vehículos; dicha dinámica ha venido sucediendo de forma paulatina desde 2004, como se muestran en las gráficas 1 y 2.

3 De acuerdo con el Ministerio de Transporte (s. f.) todo vehículo de servicio público para transporte de pasajeros debe tener una tarjeta de operación que tiene vigencia por dos (2) años, excepto en el caso del servicio individual que es de un año (1) año. Dicha tarjeta la debe gestionar la empresa a la cual se encuentra vinculado el vehículo ante la autoridad de transporte competente con dos (2) meses de anticipación a su vencimiento. 
Laura L. Amézquita - Duván F. Durán Matiz - Danny Heriberth Fajardo Morales

Tabla 1. Composición parque automotor del TPC, 2011-2012

\begin{tabular}{|c|c|c|c|c|c|c|c|}
\hline & \multicolumn{2}{|c|}{ CON TO } & \multicolumn{2}{c|}{ SIN TO } & \multicolumn{3}{c|}{ TOTAL } \\
\hline TIPO & 2011 & 2012 & 2011 & 2012 & 2011 & 2012 & $\Delta \%$ \\
\hline BUS & 6.455 & 5.574 & 780 & 403 & 7.235 & 5.977 & $-17,4 \%$ \\
\hline BUSETA & 3.572 & 2.626 & 376 & 426 & 3.948 & 3.052 & $-22,7 \%$ \\
\hline MICROBUS & 4.667 & 4.418 & 198 & 141 & 4.865 & 4.559 & $-6,3 \%$ \\
\hline & 14.694 & 12.618 & 1354 & 970 & 16.048 & 13.588 & $-15,3 \%$ \\
\hline
\end{tabular}

Fuente: elaboración propia con información Steer Davies Gleave (2012) y Registro Distrital Automotor (RDA)Concesión Servicios Integrales para la Movilidad (SIM). Cálculos Dirección de Estudios Sectoriales y de Servicios - SDM.

Gráfica 1. Tipo de vehículo de TPC con T0, 2011-2012

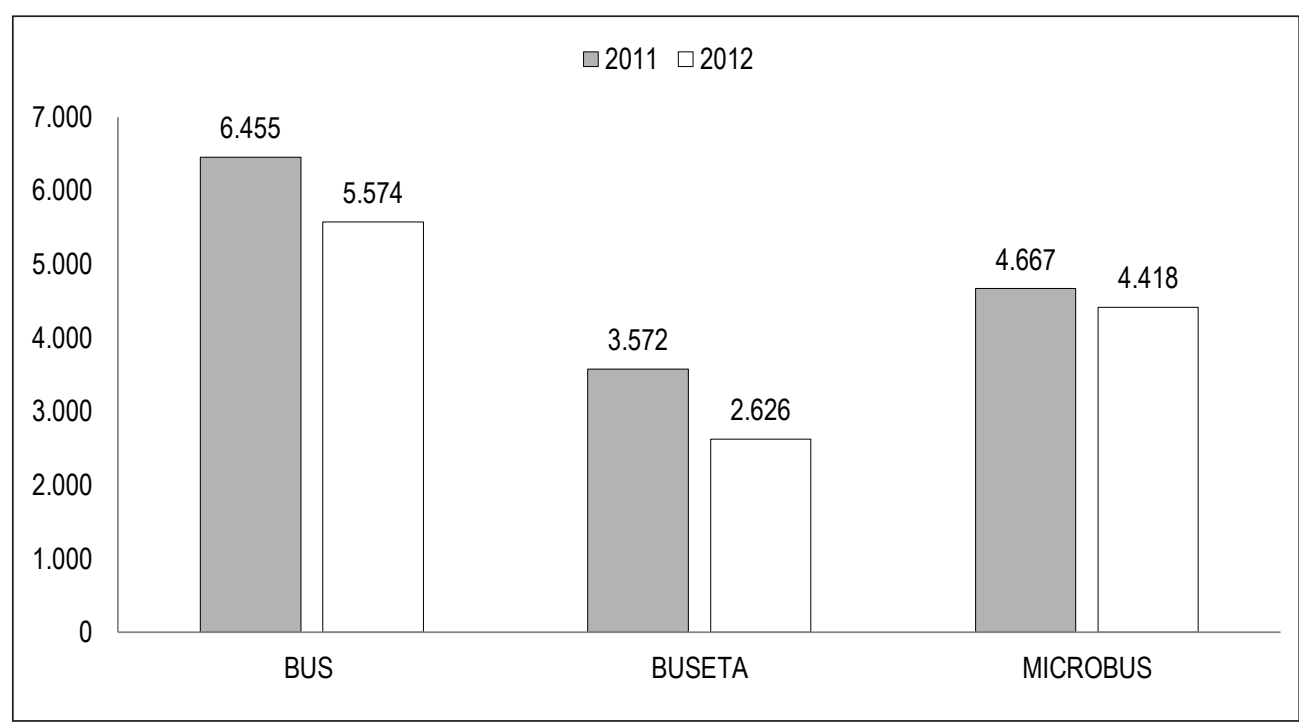

Fuente: elaboración propia con información de Steer Davies Gleave (2012).

El problema de movilidad que representa el TPC en Bogotá es la competencia y no complementariedad con otros modos de transporte, incluyendo el Transmilenio. Los esquemas de gestión y operación propuestos desde la academia como desde la autoridad local enfrentan la discusión sobre la eficiencia en el uso monopólico de infraestructuras urbanas, así como la puja de los dueños (privados) y operadores del servicio, cuyo interés de maximización de beneficios no es concordante con el objetivo de la disminución de externalidades negativas de las que terminan siendo afectados los usuarios del transporte público. 
Matriz origen-destino y eficiencia en modos de transporte urbano: un análisis de la movilidad de Bogotá

Gráfica 2. Comportamiento mensual del parque automotor de transporte público colectivo urbano activo en Bogotá año 2012

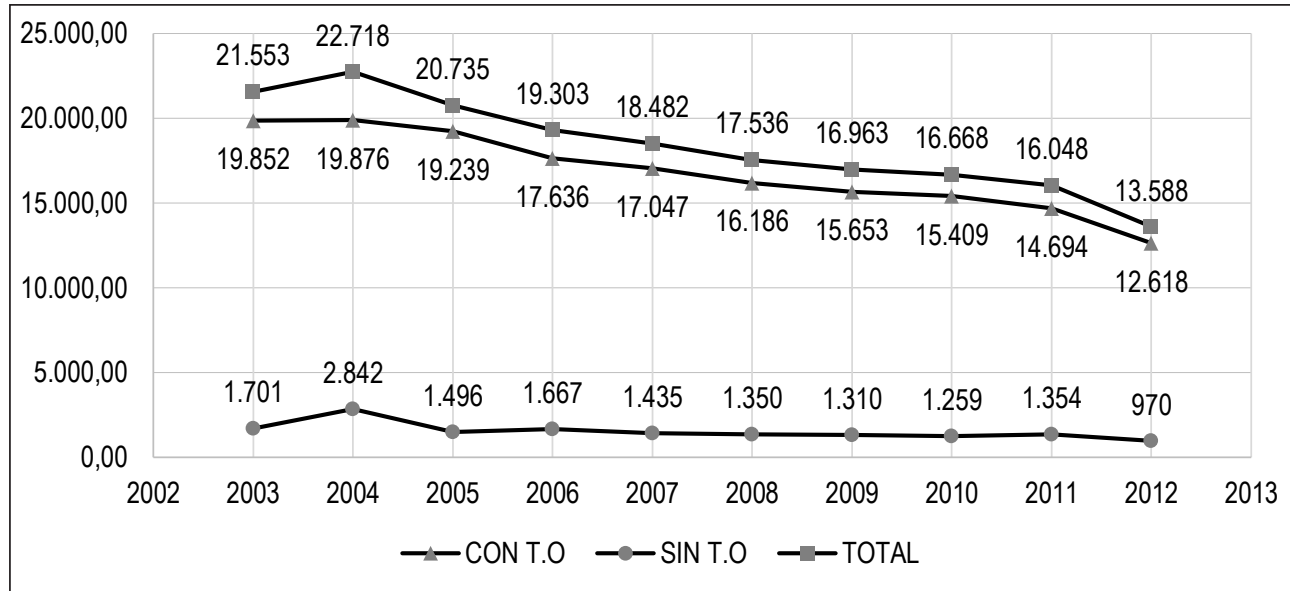

Fuente: Elaboración propia con información de registro distrital automotor (RDA) - Concesión servicios integrales para la movilidad (SIM). Cálculos dirección de estudios sectoriales y de servicios - SDM.

Según Rojas y Mello (2005, p. 108):

De forma directa a través de las administraciones locales, con dirección y acción propia del sector público o de forma indirecta por la constitución de órganos, empresas y sociedades públicas o mixtas, por parte de la administración local. Otra posibilidad es la delegación a terceros sin la intervención directa o indirecta de órganos públicos o la gestión y operación de orden nacional y público, a través de una única entidad nacional o regional que se encarga de todas las funciones.

El modelo que aplica el TPC en Bogotá consiste en que las empresas privadas de transporte público funcionan bajo el esquema de afiliación de los vehículos prestadores del servicio para la respectiva operación, es decir, que no actúan como administradoras del parque automotor; por el contrario, actúan como empresas afiladoras que incorporan los vehículos al sistema. De esta manera los vehículos que son propiedad de terceros operan en las rutas autorizadas por la Secretaría Distrital de Movilidad a las empresas afiladoras, a través de expedición de resolu ciones que no implican una relación contractual entre la ciudad y el operador, sino que se constituyen como una licencia bajo un acuerdo especificado. Esta situación se ilustra de manera simplificada en el esquema 1. 
Esquema 1. Estructura empresarial y la "guerra del centavo"

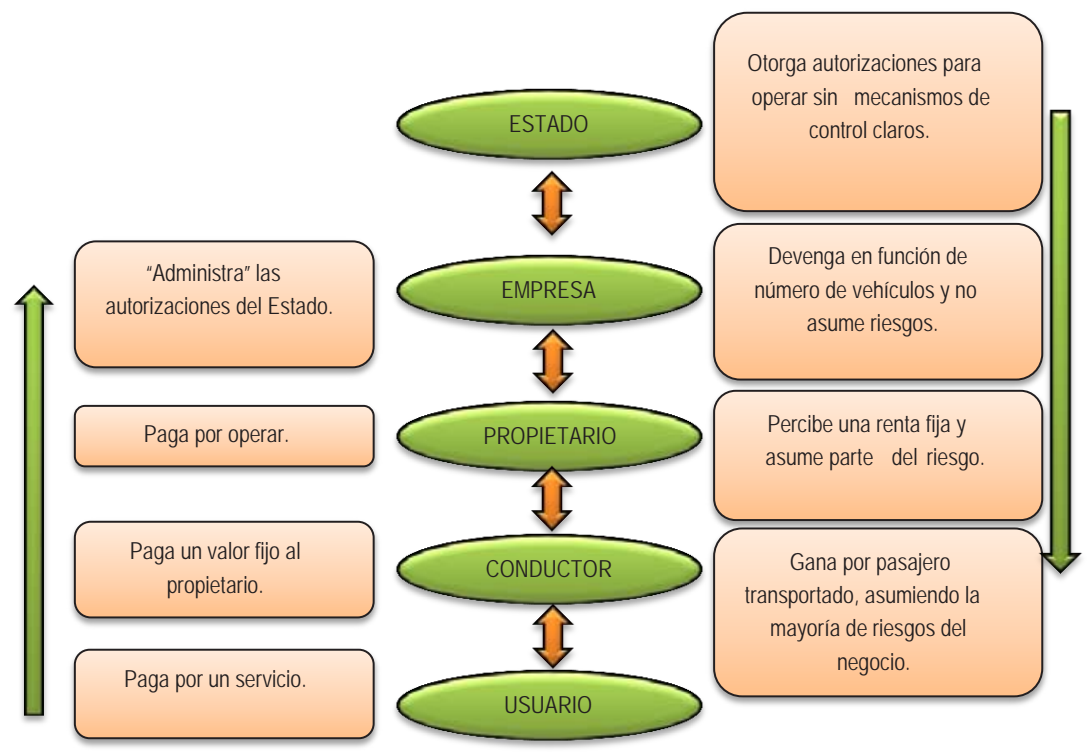

Fuente: Tomado del DNP (2003, p. 7)

La "guerra del centavo" que describe el DNP (2003) evidencia la competencia entre los operadores por obtener mayores ganancias, la cual no genera estímulos a los operadores para mejorar el servicio. Por ejemplo, recoger un mayor número de usuarios en las vías sin paraderos fijos permite un servicio ineficiente y de baja calidad. De forma clara esta, entre muchas prácticas, constituye una externalidad negativa que propicia la ejecución desordenada del sistema e impide aprovechar el espacio público (malla vial) de una manera adecuada.

Para el año 2012 el TPC no contaba con vías exclusivas para su operación, a diferencia de Transmilenio, que según el IDU (2012) contiene el 6,6 \% de la malla vial correspondiente a $1030 \mathrm{Km}$-carril para uso individual. Para el mismo año el TPC circulaba por los diferentes corredores viales de la ciudad equivalentes al $93.4 \%$ restantes (14.529 Km-carril) junto con vehículos de transporte privado, taxis y los buses del SITP.

Para Alfonso (2013) la sobreoferta de vehículos, como la prolongación secular de los recorridos y el aumento en tiempos de viaje contribuyen a una movilidad entrópica de la ciudad. El problema de movilidad entrópica, describe Alfonso (2013, p. 167) se da "debido a que una porción considerable de la energía empleada a diario en la movilización de los residentes en la ciudad se dilapida, puesto que no produce riqueza alguna y, 
Matriz origen-destino y eficiencia en modos de transporte urbano: un análisis de la movilidad de Bogotá

por el contrario, origina un conjunto de efectos socialmente indeseables". En sentido estricto, proliferan los generadores de una movilidad problemática que se encauzan en la creación de un factor determinante representado en el incremento de los tiempos de viaje. De esta manera Alfonso (2013) señala que la flexibilización del contrato de trabajo, el rezago vial, el abuso en el uso del vehículo particular y la ingobernabilidad en la vía se resumen en el incremento de los tiempos de viaje.

\section{METODOLOGÍA Y ANÁLISIS DE RESULTADOS}

A partir del objetivo de indagar la forma como los habitantes de una zona metropolitana se movilizan -en esencia los bogotanos y habitantes de las poblaciones ubicadas en las zonas periféricas de la ciudad ${ }^{4}$-, se trataron los datos de la encuesta de movilidad 2011, elaborada por la unión temporal Steer Davies Gleave y el Centro Nacional de Consultoría para la Secretaría Distrital de Movilidad de Bogotá5. A continuación, se presentan las matrices origen-destino y se estudia la eficiencia en los tiempos de viaje en función de la distancia y los modos de transporte, en especial TPC y Transmilenio.

Las zonas de análisis de transporte (ZAT) constituyen una división particular e innovadora en el estudio de las dinámicas de la ciudad. Para el año 2007 Bogotá contaba con una propuesta por parte de Cal E Mayor y asociados de 767 ZAT internas, más 57 zonas externas en los municipios de Cundinamarca ${ }^{6}$ para un total de 824 zonas de transporte, de acuerdo con Steer Davies Gleave (2012, p. 11). Posteriormente la firma Steer Davies Gleave, para el año 2011, propone 39 zonas nuevas en los 17 municipios vecinos (ver anexo A).

Con base en la división zonal propuesta por la consultora, las ZAT tienen características particulares desde el ámbito económico, social, de accesibilidad (transporte), estratificación, uso del suelo, tamaño, etc., en las cuales se tuvo en cuenta, en orden de prioridad, la zonificación con base en sectores censales, ya que permite establecer modelos de desplazamiento de origen-destino (OD). De igual manera, se tiene en cuenta el estrato socio-económico que, aunque no refleja de

4 Los municipios de Soacha, Bojacá, Cajicá, Chía, Cota, Facatativá, Funza, Gachancipá, La Calera, Mosquera, Sibaté, Sopó, Tabio, Tenjo, Tocancipá y Zipaquirá, de acuerdo con lo establecido en el contrato de encuesta de movilidad 2011 con la consultora Steer Davies Gleave.

5 La información se divide en cuatro módulos de los cuales el último presenta una base adicional cuyos datos se encuentran imputados (el termino se refiere al método que remplaza cada valor de ítems "norespuesta" con un valor disponible de un encuestado aleatorio de características similares dentro del mismo estudio . La estructura del módulo D imputados (referente a los desplazamientos realizados por las personas del hogar) fue trabajada para obtener los diversos modos de transporte que las personas utilizan para llevar a cabo sus diferentes actividades.

6 Según Steer Davies Gleave (2012) para los 17 municipios vecinos a Bogotá las zonas de transporte correspondían a una por municipio, a excepción de Soacha la cual tiene subdivisión interna e incorpora el municipio de Granada. Las demás zonas externas agrupan varios municipio. 


\section{ZONAS DE ANALISIS DE TRANSPORTE - VIAJES ORIGEN}

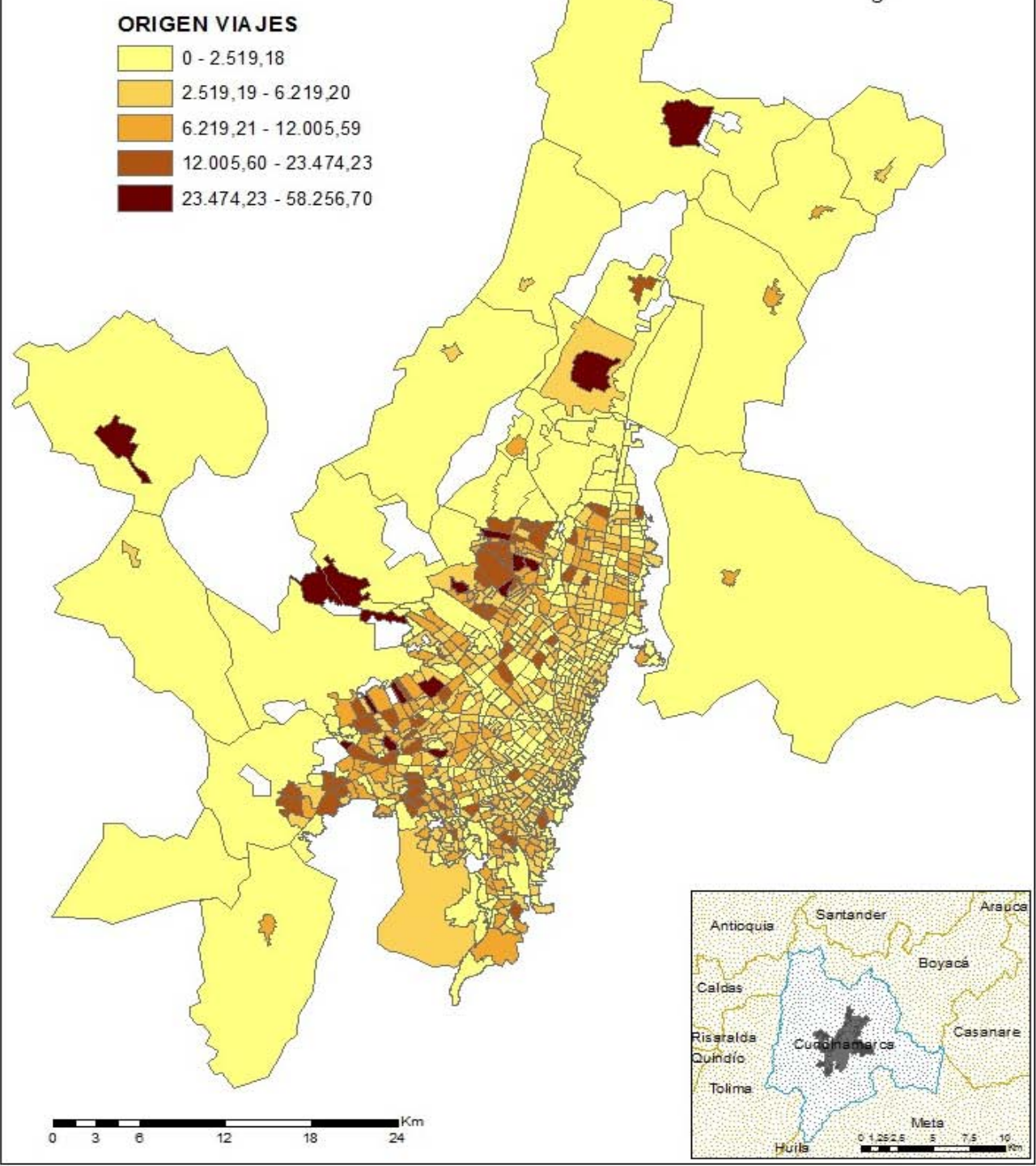

Fuente: elaboración propia con información de la encuesta 2011 de la Secretaría de Movilidad 
Matriz origen-destino y eficiencia en modos de transporte urbano: un análisis de la movilidad de Bogotá

Mapa 2. Viajes de destino. Zonas de análisis de transporte

\section{ZONAS DE ANALISIS DE TRANSPORTE - VIAJES DESTINO}

\section{DESTINO VIA JES}
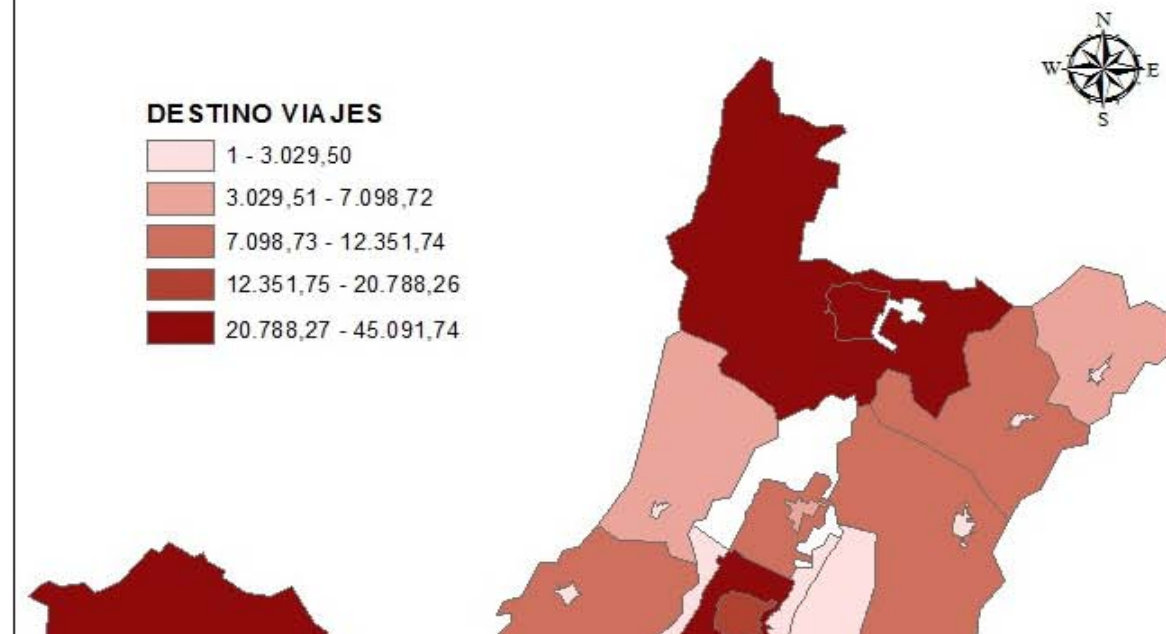

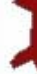
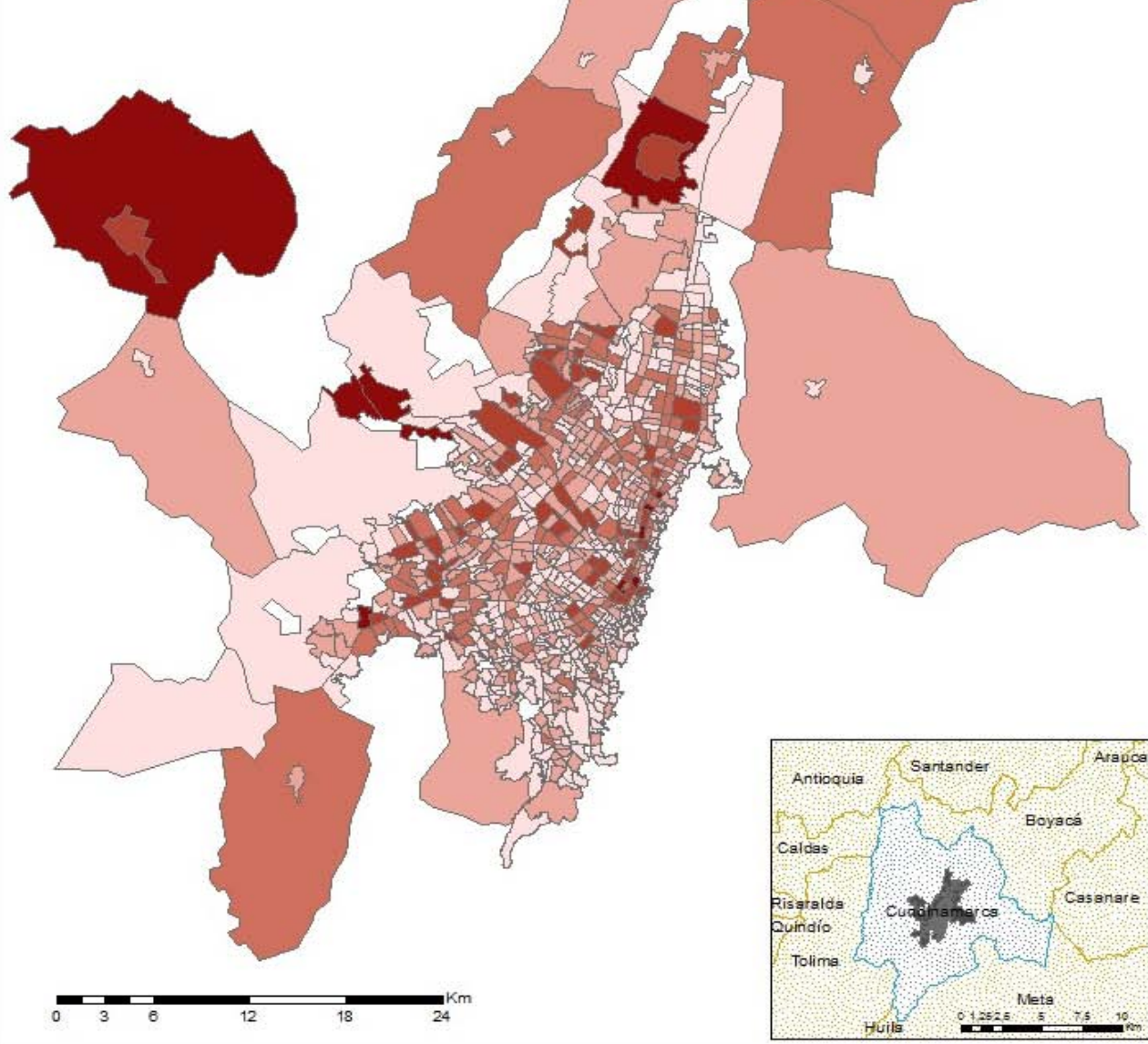

Fuente: elaboración propia con información de la encuesta 2011 de la Secretaría de Movilidad 
manera consistente el nivel de ingreso de la población, permite tener un punto de partida para la clasificación de hogares y división de la ciudad.

Los mapas 1 y 2 muestran la totalidad de viajes con origen y destino que, en consecuencia, tienen una escala de colores contrario. Es importante mencionar que la división por ZAT contiene variables por motivo de viaje de las personas, dentro de la cuales se encuentran: búsqueda de trabajo, actividades de trabajo, estudio y recreación. Se presentan dichos motivos que fueron seleccionados por el mayor peso que poseen dentro de la base de datos; adicionalmente, porque son características fundamentales para el proceso productivo de la ciudad y su zona periférica.

De esta manera el análisis espacial expone que para la zona periférica y la periurbana de la ciudad, se obtiene el mayor número de viajes por origen, es decir, que zonas comprendidas dentro de localidades como Suba, Engativá, Fontibón, Kennedy, Bosa, Ciudad Bolívar y Usme constituyen el punto de partida de viajes que realizan las personas para actividades como trabajo, estudio y recreación. Del mismo modo, para los municipios de Zipaquirá, Chía, Funza y Soacha se observa una dinámica de mayor cantidad de viajes por origen. Por otro lado, en la misma referencia espacial de viajes-origen, se identifica que la zona del centro de Bogotá está caracterizada por una menor cantidad de viajes, lo que podría significar una dinámica monocentrista, es decir, que debido a la fuerte concentración productiva los viajes destino tendrían una mayor relevancia en esta zona.

Dicho argumento puede expresarse en el análisis espacial de viajes-destino, ya que localidades como Santa Fe, Chapinero y Los Mártires representan una mayor cantidad de viajes-destino reflejados por una alta concentración de entidades bancarias, zonas comerciales, instituciones públicas e inclusive instituciones académicas como universidades que brindan una visión clara de esta dinámica. De igual manera, para los municipios aledaños como Facatativá, Chía y Funza se observa una fuerte dinámica de destino de los viajes. Esto se explica debido a las cabeceras municipales, es decir, las personas que viven en la zona periurbana realizan el viaje a las zonas rurales de cada municipio.

En este orden de ideas, se presentan las matrices origen-destino para Bogotá dentro de las cuales se integraron las ZAT por localidades; también se incluyó la población de Soacha debido a que está conurbada con Bogotá y en todo sentido presenta una relación estrecha con la capital7 ${ }^{7}$ La gráfica 3 ratifica la concentración

7 De igual manera se realizó la matriz origen-destino de Bogotá y los municipios incluidos en la encuesta, mostrando como resultado una notable diferencia en la cantidad de los viajes que se realizan Bogotá-Bogotá frente a las demás combinaciones con los municipios aledaños; no es sorprende por los registros de la encuesta, centrada en Bogotá, sin embargo, se puede consultar en el Anexo B. 
como destinos que tienen las localidades de Chapinero, Usaquén, Santa Fe y Los Mártires, y la concentración como origen de Soacha, Kennedy, Ciudad Bolívar, Usme, Engativá, Fontibón y Bosa.

\section{Gráfica 3. Matriz origen-destino. Localidades de Bogotá y Soacha}

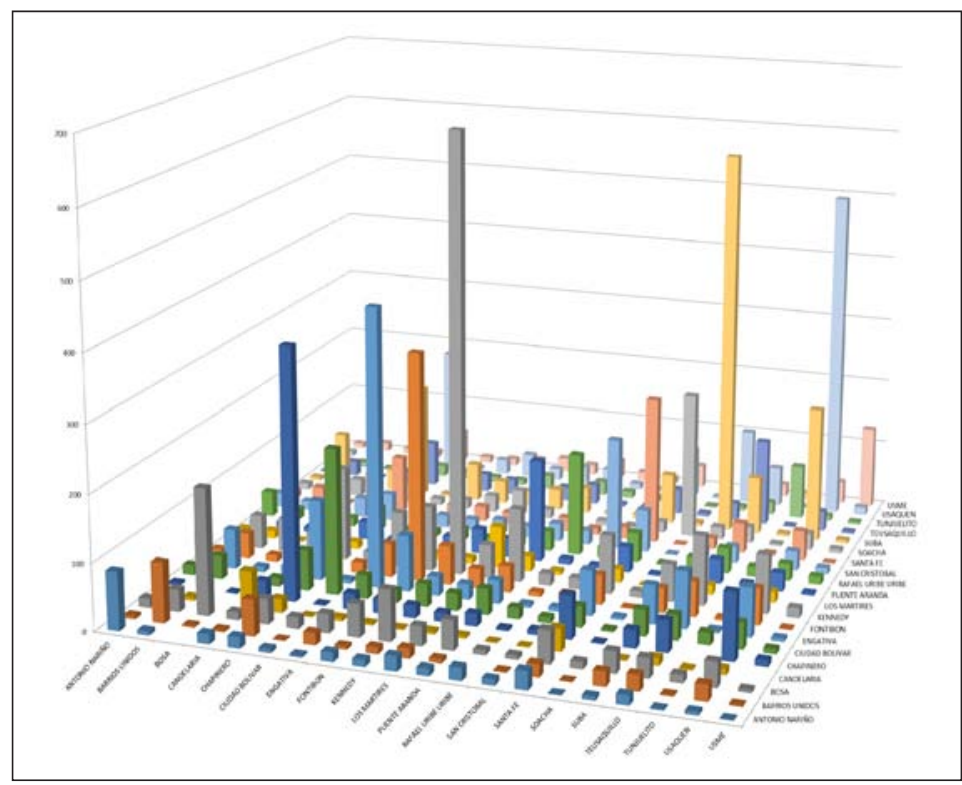

Fuente: elaboración propia con información de la encuesta 2011 de la Secretaría de Movilidad.

Para hacer una aproximación a la comprensión del problema de movilidad en Bogotá, se propone un modelo de regresión lineal simple, en corte transversal con los datos que la encuesta de movilidad 2011 permite cuantificar ${ }^{8}$. Se aplican variables tales como tiempos de viaje (TV), distancia proyectada en cada viaje entre ZAT (DV) y el modo de transporte en el que se movilizan las personas (M) ${ }^{9}$, datos que se resumen en la tabla 2.

8 La información disponible en la Encuesta tiene procedencia en preguntas son de orden categórico (no numéricas). Las dos variables, únicas posibles de cuantificar se construyeron así: Los tiempos de viaje fueron inferidos de las preguntas: hora de inicio del viaje y hora de fin del viaje (calculada en minutos, sin distinción entre horas pico y horas valle), y las distancias fueron calculadas de acuerdo al registro de origen y de destino por ZAT.

9 Los modos de transporte constituyen una variable tipo dummy (dicotómica). 
Tabla 2. Variables incluidas en el modelo

\begin{tabular}{|l|l|}
\hline VARIABLE DEPENDIENTE & Tiempo de viaje (TV) \\
\hline VARIABLE INDEPENDIENTE 1 & Distancia proyectada (DV) \\
\hline VARIABLE INDEPENDIENTE 2 & $\begin{array}{l}\text { Modo (M) } \\
\text { - Transmilenio: (dummytm) } \\
\text { - TPC: (dummytpc) }\end{array}$ \\
\hline
\end{tabular}

Fuente: elaboración propia.

Se especifica, entonces, la siguiente función: . Se toman 77.715 observaciones (sin valores perdidos) que constituyen la muestra de los viajes OD que tiene la encuesta. Los resultados obtenidos se presentan en la tabla $3^{10}$.

Estos resultados muestran que los usuarios de Transmilenio tienden a demorarse más tiempo en los viajes que los usuarios de TPC; si bien las dos variables van en la misma dirección el coeficiente (estimado) de TPC es un poco mayor. Asimismo, ambas variables dicotómicas son significativas y no tienen problemas de multicolinealidad como lo refleja la prueba VIF ${ }^{11}$.

Tabla 3. Resultados del modelo

\begin{tabular}{|c|c|}
\hline & TV \\
\hline \multirow{2}{*}{ DV } & 0,001 \\
\hline & $(94,47)^{* *}$ \\
\hline \multirow{2}{*}{ Dummytpc } & 33.209 \\
\hline & $(107,20)^{* *}$ \\
\hline \multirow{2}{*}{ Dummytm } & 40.803 \\
\hline & $(87,62)^{* *}$ \\
\hline \multirow{2}{*}{ Constant } & 23.512 \\
\hline & $(139,86)^{* *}$ \\
\hline Observations & 77.715 \\
\hline R-squared & 0,29 \\
\hline \multicolumn{2}{|c|}{$\begin{array}{l}\text { Absolute value of t-statistics in parentheses } \\
\text { * significant at } 5 \% \text { level; }{ }^{* *} \text { significant at } 1 \% \text { level }\end{array}$} \\
\hline
\end{tabular}

Fuente: elaboración propia

10 Se corre la regresión con la herramienta "robust" que "Se produce estimadores para datos comunes (cada observación independiente), los datos agrupados (datos no independientes dentro de los grupos, pero independientes entre los grupos), y complejos datos de la encuesta de una etapa muestreo por conglomerados de estratificado". Dado que a través de la prueba de White (White's general test statistic :1.412,777 Chi-sq (6) P-value $=4 . e-302$ ) se observó problemas de heterocedasticidad.

11 Factor de inflación de varianza por sus siglas en inglés. Con una media de 1,05 para la regresión (1,06 para dummytm; 1,05 para dv, y 1,04 para dummytpc). 


\section{CONCLUSIONES Y CONSIDERACIONES FINALES}

La problemática de la movilidad en la ciudad de Bogotá obliga a estudiar los modos de desplazamiento y los tiempos de duración de los mismos. El TPC representa el modo de transporte tradicional, cuya operación se lleva a cabo por entidades privadas que están suscritas a la Secretaría de Movilidad para la prestación del servicio. El objetivo principal de estas empresas es el desplazamiento de pasajeros a través de la malla vial de la ciudad, pues obtienen más ganancias por un mayor número de usuarios. Debido a su naturaleza como agentes privados y a su objetivo de maximización de beneficios por el recaudo, se refleja una operación desordenada o entrópica que ocasiona externalidades a la movilidad en general, y afecta variables como los tiempos de viaje de las personas. De igual manera, la inadecuada infraestructura para la ejecución de operación ha permitido que los diferentes automotores (bus, buseta o microbús) realicen las paradas en cualquier lugar sobre la vía, lo que ocasiona congestión vehicular y se sufre en la ciudad lo que los expertos han denominado ingobernabilidad en la vía.

De forma paralela funciona un sistema tipo BRT, Transmilenio, que surgió como una alternativa de transporte masivo con el propósito de organizar la movilidad de la ciudad. Este sistema está caracterizado por tener vías exclusivas y una infraestructura definida que le ha permitido tener controles y, en consecuencia, ser más coordinado en la ejecución, en comparación con el TPC. Sin embargo, de acuerdo con los datos obtenidos, los viajes en Bogotá se realizan en mayor cantidad en los buses del TPC.

La localización se centra en el estudio de la forma como las personas se agrupan alrededor de un centro económico, en general el principal escenario de acumulación de capital, lo cual es evidenciado en las matrices origen-destino expuestas previamente, ya que reflejan una dinámica en donde se plantea la misma teoría de la localización al tener una mayor concentración en el origen de los viajes en las zonas periféricas y periurbanas de la ciudad y en coherencia una concentración en el destino de viajes hacia las zonas centrales de la ciudad.

Por otra parte, el modelo econométrico explicó la duración de tiempos de viajes con las distancias (lo cual es previsible); a la vez mostró que tanto el TPC como el Transmilenio afectan la duración del viaje. Infortunadamente los datos de la encuesta no permiten obtener evidencias concluyentes sobre otras variables introducidas en el modelo, los datos presentados se encuentran agregados bajo la lógica de la división político administrativa de la zona metropolitana (diferentes a la original de las ZAT). 


\section{BIBLIOGRAFÍA}

Alcaldía Mayor de Bogotá (s. f.) Formulación del Plan Maestro de Movilidad de Bogotá D.C. que incluye ordenamiento de estacionamiento. Bogotá D. C., 80p

Alcántara E. (2010). Análisis de la movilidad urbana, espacio, medio ambiente y equidad. (CAF, Ed.) Bogotá, Colombia, 204p.

Alfonso, O. (2013). El sistema entrópico de movilidad cotidiana en la zona metropolitana de Bogotá. En: Territorios, N. ${ }^{\circ} 28$, pp. 165-185.

Alonso, M. (1964). Location and Land Use. Cambridge, Harvad University Press, 240p.

Analistas Económicos de Andalucía (s. f.). El transporte: importancia económica y social. [En líneal Analistas Económicos de Andalucía, España, 39p.

Camagni, R. (2005). Economía urbana. Barcelona: Antoni Bosch Editor. 55p.

Carrasco, I. y Castaño, S. (2012). La nueva economía institucional. En: Nuevas Corrientes de Pensamiento economico ICE, N. ${ }^{\circ} 865$, pp. 43-53.

DNP -Departamento Nacional de Planeación- (2003). Documento Conpes 3260: Política Nacional de Transporte Masivo. Consejo Nacional de Política Económica y Social. Departamento Nacional de Planeación, 37p.

De Rus, G., Campos, J., y Nombela, G. (2003). Economía del Transporte, 20p.

Díaz, M. y Jimenez , F. (2002). Transportes y movilidad: ¿necesidades diferenciales según género?. Segundo Seminario Internacional sobre Género y Urbanismo Infraestructuras para la Vida Cotidiana ETSAM, Madrid, 19p.

Duch, N. (s. f.). La teoría de la localización. Barcelona: Universitat de Barcelona, 73p.

Fujita, M., Krugman, P., y Venables, A. J. (1999). The Spatial Economy. Massachusetts: Editorial Ariel, S. A., 382p.

Hernández, J. A., y Beltrán, J. G. (2010). Análisis e incidencia del gasto en transporte público en los hogares en las Localidades de Usme y Ciudad Bolívar. En: Equidad y Desarrollo, No 13, pp. 99-117.

IDU -Instituto de Desarrollo Urbano- (2012). Mantenimiento IDU Instituto de Desarrollo Urbano. Bogotá: Instituto de Desarrollo Urbano.

Matas, A. (2004). Políticas de transporte y congestión en áreas urbanas: un panorma. En: Urban Public Economics Review, N.1, pp. 63-91.

Ministerio de Transporte. (s.f.). Preguntas Frecuentes. Ministerio de Transporte de Colombia.

Moro, B. (2007). El futuro de la movilidad en Bogotá: reflexiones a propósito del Plan Maestro de Movilidad y Estacionamiento. Bogotá: Volumen 1 Cuadernos de Informe de Desarrollo Humano para Bogotá, Editor El Tiempo y Alcaldía Mayor de Bogotá, 118p.

North, D. (1990). Instituciones, cambio institucional y desempeño económico, México, Fondo de Cultura Económica, 190p. 
Rodríguez, E. (1990). Los movimientos cotidianos de la población por motivos laborales en las ciudades pequeñas de servicio: el caso de Ciudad Real. En: Cuadernos de Estudios Manchegos, No. 21, pp. 151-168.

Rojas, F. y Mello, C. (2005). El transporte público colectivo en Curitiba y Bogotá. En: Revista de Ingeniería. No. 21, Facultad de Ingeniería Universidad de los Andes, pp.106- $\overline{115}$.

SDP -Secretaría Distrital de Planeación - (2014). Región Metropolitana de Bogotá: Una visión de la ocupación del suelo. Bogotá D. C., Alcaldía de Bogotá, 442p.

Steer Davies Gleave. (2012). Encuesta de Movilidad para Bogotá 2011 Tomo II Informe final consultoría. Bogotá, Union temporal Steer Davies Gleave.

Thompson, J. (1976). Teoría económica del transporte. Curso de Economía Moderna. Madrid: Alianza Editorial, 301p.

Transmilenio (2013). Historia. Transmilenio. 


\section{ANEXOS}

\section{Anexo A. Zonificación Steer Davies Gleave 2011}

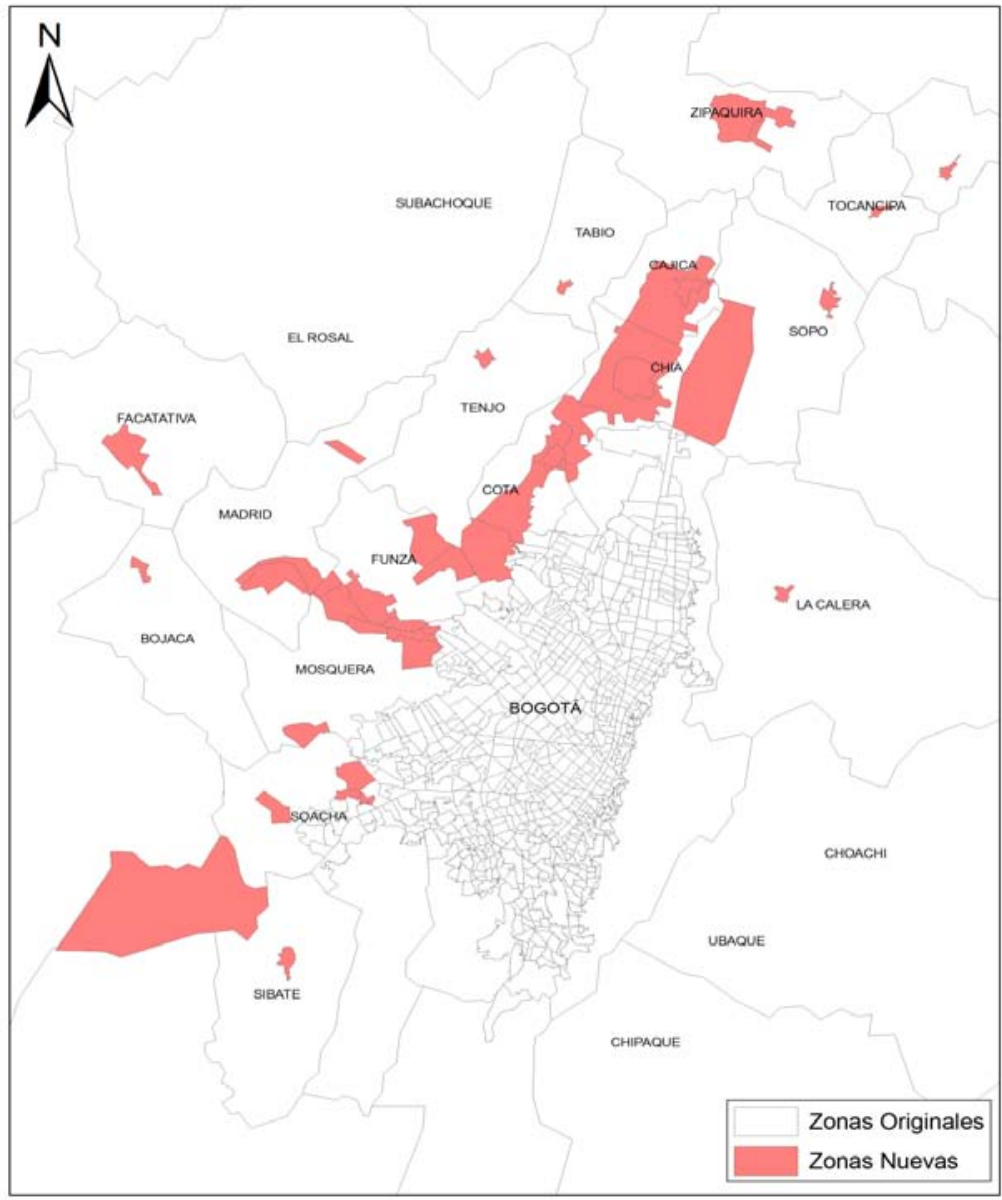

Fuente: tomado de Steer Davies Gleave ( 2012, p. 6) 
Matriz origen-destino y eficiencia en modos de transporte urbano: un análisis de la movilidad de Bogotá

Anexo B. Matriz origen-destino Bogotá y municipios aledaños

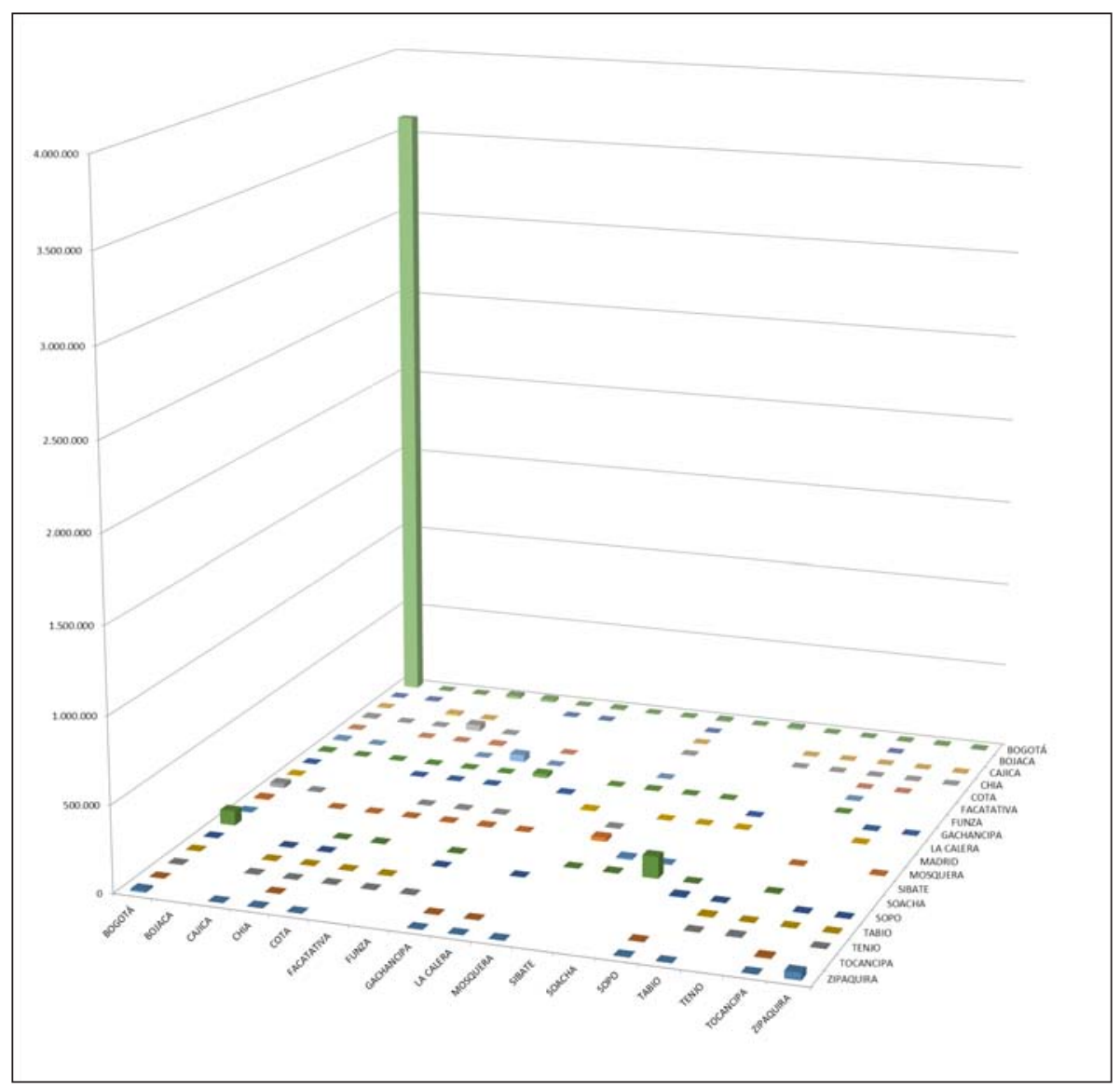

Fuente: elaboración propia con información de la encuesta 2011 de la Secretaría de Movilidad 
